\title{
Weil jede Dosis zählt: Risiken bei der Covid-19-Impfung
}

\section{LANDES GESUNDHEITS}

Massenimpfungen zu organisieren ist an sich schon eine Herausforderung. Dabei die besonderen Bedürfnisse der Covid-19-Impfstoffe zu berücksichtigen, bedarf einer umfassenden Risikobetrachtung. So geschehen im Universitätsklinikum St. Pölten, im Rahmen der Impfung von Mitarbeitern und Risikopatienten.

Claudia Wunder, Alexander Taschauer

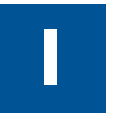

m Zusammenspiel aller Berufsgruppen ist es im Universitätsklinikum St. Pölten binnen weniger Tage gelungen, eine Impfstraße aufzubauen, die Aufklärung, Dokumentation, Gebrauchsfertigmachen und Applikation an einer Stelle vereint. Durch mobile Wände abgetrennt befindet sich dort der Arbeitsbereich der Apotheke, ausgestattet mit einem Kühlschrank und zwei aseptischen Arbeitsplätzen mit Laminar Air Flow. In Zusammenarbeit zwischen Apotheke und ärztlicher Direktion wurde eine Risikoanalyse des Gesamtprozesses in Form einer FMEA (Failure Mode and Effect Analysis) ausgearbeitet, um potenzielle Fehlerquellen zu erkennen und in weiterer Folge Maßnahmen zur Fehlervermeidung zu setzen (Tabelle 1). 


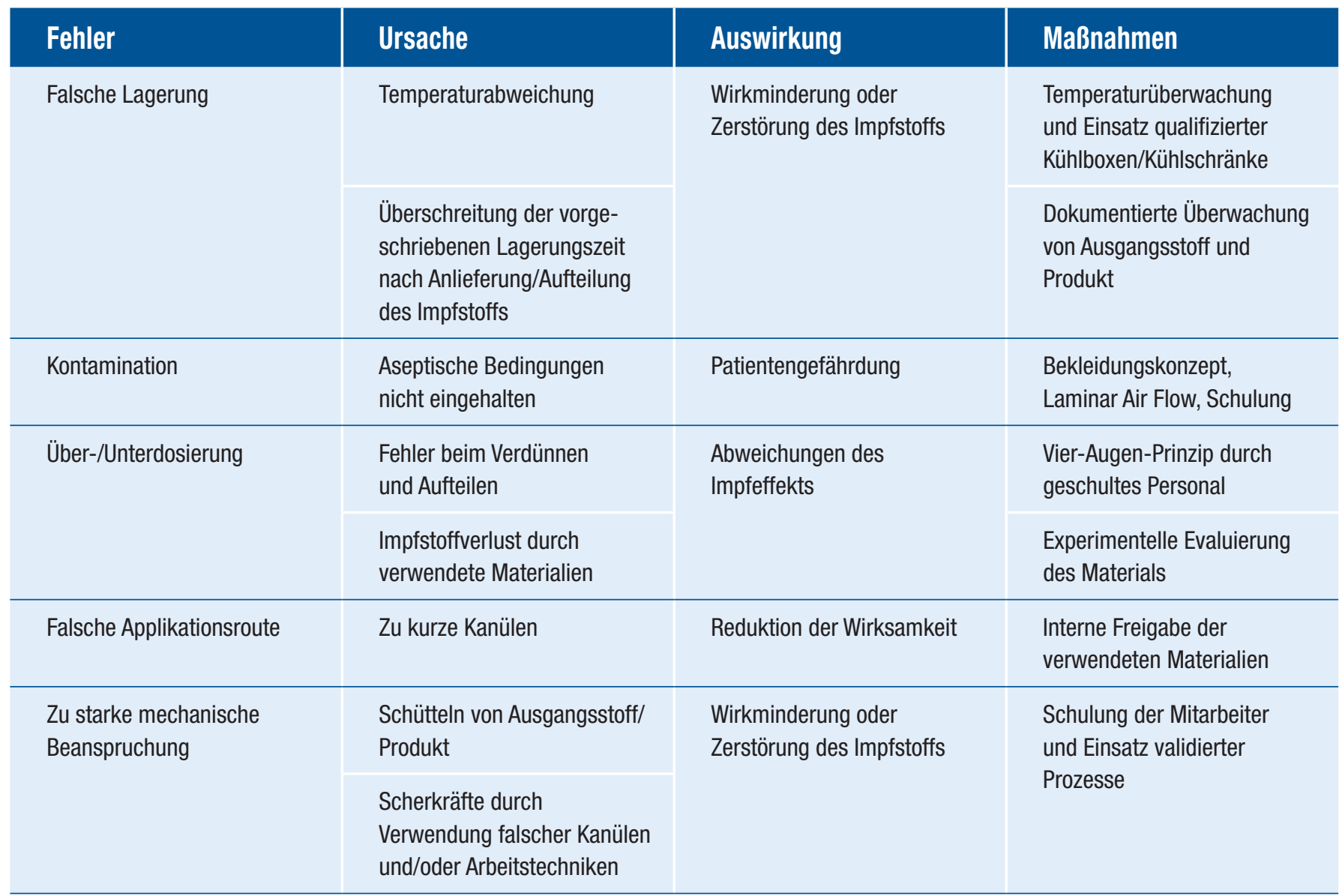

Tab. 1: Potenzielle Risiken

Startpunkt der Überlegungen war die Auseinandersetzung mit den Eigenschaften des Impfstoffs der Firma Pfizer/BioNTech, der als erstes zur Verfügung stand.

Einer hohen Impfeffizienz steht eine hohe Empfindlichkeit sowohl chemischer als auch physikalischer Natur gegenüber. Diese Erkenntnisse wurden auf den Umgang mit den später verfügbaren Impfstoffen der Firmen Moderna und AstraZeneca ausgeweitet. Die resultierenden Herstellvorschriften werden kontinuierlich an den aktuellen Wissensstand angepasst. Erfahrenes Apothekenpersonal garantiert die Einhaltung der Spezifikationen. Die Mitarbeiter werden gemäß den Vorgaben des pharmazeutischen Qualitätsmanagementsystems laufend geschult.
Die Berücksichtigung aller Risikofaktoren garantiert einerseits, dass im Rahmen der Verabreichung keine Wirkminderung oder Patientengefährdung eintritt. Andererseits wird die optimale Verwertung des Inhalts jeder Durchstechflasche gesichert, was besonders in der derzeitigen Situation der allgemeinen Knappheit an Covid-19-Impfstoffen von Relevanz ist.

Bestätigt wurde die Notwendigkeit einer Risikobetrachtung durch eine aktuelle CDC-Publikation (Centers for Disease Control and Prevention), in der sich fast $25 \%$ der eingegangenen Meldungen auf Fehler beim Gebrauchsfertigmachen, bei der Lagerung oder beim Materialeinsatz zurückführen lassen.
Autoren:

\author{
Mag. Claudia Wunder, MBA, aHPh \\ Leitung Apotheke
}

Mag. Dr. Alexander Taschauer Qualitätsmanagementbeauftragter

Universitätsklinikum St. Pölten NÖ Landesgesundheitsagentur www.landesgesundheitsagentur.at 